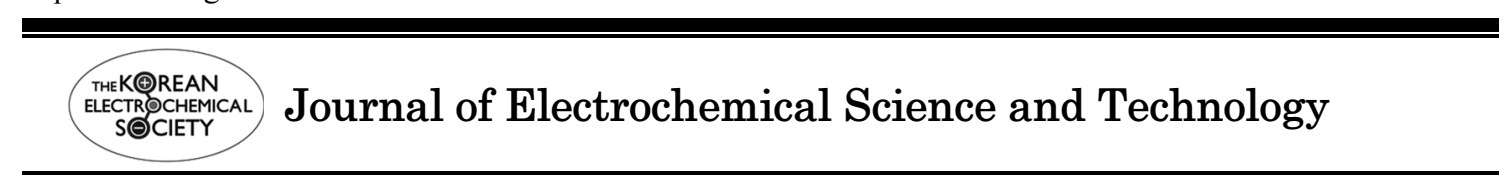

\title{
Paper-Based Bipolar Electrochemistry
}

\author{
Christophe Renault, Karen Scida, Kyle N. Knust, Stephen E. Fosdick, and Richard M. Crooks* \\ Department of Chemistry and the Center for Nano- and Molecular Science and Technology, The University of Texas at Austin, \\ 105 E. 24th St. Stop A5300, Austin, TX 78712-1224
}

\begin{abstract}
:
We demonstrate that carbon electrodes screen-printed directly on cellulose paper can be employed to perform bipolar electrochemistry. In addition, an array of 18 screen-printed bipolar electrodes (BPEs) can be simultaneously controlled using a single pair of driving electrodes. The electrochemical state of the BPEs is read-out using electrogenerated chemiluminescence. These results are important because they demonstrate the feasibility of coupling bipolar electrochemistry to microfluidic paperbased analytical devices ( $\mu$ PADs) to perform highly multiplexed, low-cost measurements.
\end{abstract}

Keywords: Bipolar electrochemistry, Paper-based microfluidic analytical device, Electrogenerated chemiluminescence, Screen-printed electrode.

Received December 9, 2013 : Accepted December 27, 2013

\section{Introduction}

Here, we demonstrate that bipolar electrochemistry can be implemented on paper substrates and read out by optical signal transduction. Specifically, we show that simple and inexpensive screen-printed carbon electrodes can be used as bipolar electrodes (BPEs) for microfluidic paper-based analytical devices ( $\mu$ PADs). In fact, using just a pair of driving electrodes and a simple power supply, electrogenerated chemiluminescence (ECL) can be simultaneously activated at a $\mu$ PAD containing an array of 18 BPEs. This result is important because it enables highly multiplexed electrochemical measurements on $\mu$ PADs.

Following the introduction of $\mu$ PADs by the Whitesides group, there has been considerable interest in the use of such devices for point-of-care (POC) diagnostics. ${ }^{1-5} \mu$ PADs are typically prepared by patterning hydrophobic walls (using materials like photoresist and wax) on or within materials such as cellulose paper, ${ }^{2}$ thereby forming hydrophilic channels that

*Corresponding author. Tel.: 512-475-8674

E-mail address: crooks@cm.utexas.edu direct the flow of a liquid sample to specific reaction zones. These zones are loaded with reagents that in the presence of an analyte induce either an optical ${ }^{6-7}$ or electrochemical ${ }^{8-10}$ output. In the case of electrochemical detection, electrodes can be directly integrated in the $\mu \mathrm{PAD}$ by inkjet, stencil- or screen-printing. ${ }^{8,11-12}$ Due to their ease of fabrication, screen-printed electrodes (SPEs) are commonly used to perform amperometric and potentiometric measurements on $\mu$ PADs. ${ }^{13-}$ ${ }^{16}$ SPEs have also been used to produce optical signals via ECL. $^{17}$

The time required to fabricate a single SPE is no different than the time necessary to fabricate an array; therefore, multiple SPEs can be easily integrated on a single $\mu$ PAD platform to perform multiplexed assays. However, traditional three-electrode electrochemical approaches require a direct electrical connection to each SPE for read-out, and this can be difficult to implement on a simple POC sensor. This problem can be solved using bipolar electrochemistry, because faradaic processes are driven on the SPE without a direct electrical connection to a power supply. ${ }^{18}$ Consequently, an array of BPEs can be operated using a single pair of driving electrodes and a simple power 
supply. For example, our group has shown that a dense array of 1000 BPEs can be actuated simultaneously using only two driving electrodes. ${ }^{19-20}$

The principles of bipolar electrochemistry and BPEbased sensing are discussed in several recent reviews. ${ }^{18,21}$ As shown in Scheme 1a, an externally applied potential bias $\left(E_{\mathrm{tot}}\right)$ of sufficient magnitude can result in enough potential dropped over a $\operatorname{BPE}\left(\Delta E_{\text {elec }}\right)$ to drive oxidation and reduction reactions simultaneously at opposite ends of the BPE. For example, Scheme $1 b$ shows the case in which a carbon SPE, exposed to an aqueous electrolyte solution, produces hydroxide ions at the BPE cathode and protons at the BPE anode as a result of water reduction and oxidation, respectively. A very early application of bipolar electrochemistry to paper substrates was described by Bradley et al. in $1999 .^{22}$ Specifically, they used graphite microbeads trapped within the pores of a paper substrate as individual BPEs to reduce a palladium molecular precursor and thereby obtain a metal catalyst network uniformly dispersed onto the paper structure. Several examples of sensors using BPEs microfabricated on glass substrates have also been reported in the literature. ${ }^{23-26}$ For example, our group used ECL produced by the co-oxidation of tris(bipyridine)ruthenium(II), $\mathrm{Ru}(\mathrm{bpy})_{3}{ }^{2+}$, and tri- $n$-propylamine at the BPE anode to indirectly report sensing events at the cathodic poles of BPEs. ${ }^{27-28}$

In the present article, we demonstrate that carbon SPEs are suitable for performing bipolar electrochem-

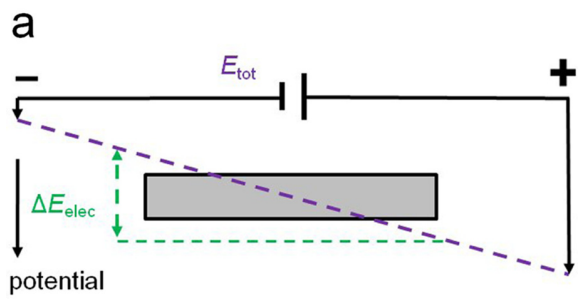

b

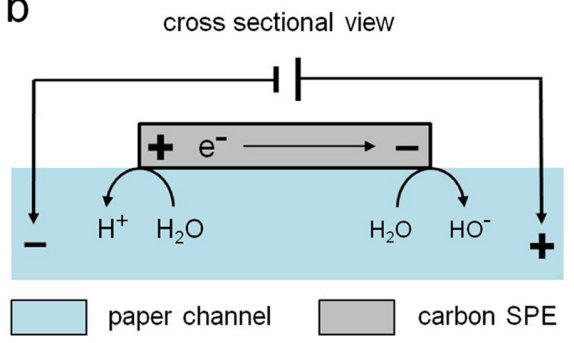

Scheme 1. istry on $\mu$ PADs and, therefore, such a platform can host a dense array of many carbon SPEs. Moreover, we show that 18 carbon SPEs can be used as BPEs and simultaneously generate ECL. The ECL signal is stable and reproducible and can be used as an optical readout. We believe that these results open new possibilities for the creation of highly multiplexed sensing systems on $\mu$ PADs.

\section{Experimental Section}

\subsection{Chemicals.}

$\mathrm{NaH}_{2} \mathrm{PO}_{4}, \mathrm{Na}_{2} \mathrm{HPO}_{4}$, and 2-(dibutylamino)ethanol (DBAE) were purchased from Sigma-Aldrich ( $\mathrm{St}$ Louis, MO). The $\mathrm{pH}$ indicator solution was purchased from Acros (Cat \# 61231-5000, Morris Plains, NJ). $\mathrm{Ru}(\text { bpy })_{3} \mathrm{Cl}_{2} \cdot 6 \mathrm{H}_{2} \mathrm{O}$ was purchased from Strem Chemicals (Newburyport, MA). $\mathrm{NaCl}$ and Whatman grade 1 chromatography paper were purchased from Fisher Scientific (Waltham, MA). Carbon ink (CI-2042) was purchased from Engineered Conductive Materials (Delaware, $\mathrm{OH}$ ). All the chemicals were used without further purification. Deionized (DI) water having a resistivity $>18 \mathrm{M} \Omega \cdot \mathrm{cm}$ was used for all experiments (Milli-Q gradient system, Millipore, Bedford, MA).

\subsection{Device fabrication.}

The patterns for the single-BPE and BPE array $\mu$ PADs are provided in Fig. 1. The devices were fabricated as follows. First, the paper channels were printed on Whatman grade 1 chromatography paper $(20 \times 20 \mathrm{~cm}$ sheet $)$ using a Xerox $8570 \mathrm{DN}$ inkjet wax printer, and then the wax/paper assembly was heated in an oven at $120^{\circ} \mathrm{C}$ for $90 \mathrm{~s}$ to form the hydrophobic wax barriers. ${ }^{29,30}$ Second, the patterns of the electrodes were photolithographically defined onto 305 thread/ in $^{2}$ mesh (Ryonet W2024305) using a screen printing kit (Ryonet, SKSTARTERKIT). Third, carbon electrodes were manually screen-printed onto the wax patterned paper using the patterned mesh as a mask. Fourth, the electrodes were dried for $15 \mathrm{~min}$ at $23^{\circ} \mathrm{C}$ and then for 30 additional minutes at $65^{\circ} \mathrm{C}$ to stabilize the electronic properties of the ink. In the present article, all the electrodes were fabricated by screen-printing and are therefore referred to as SPEs. It is important to note that these SPEs are also referred to as BPEs and driving electrodes according to their function in the device. Finally, the driving electrodes, which are only used to power the device, were con- 


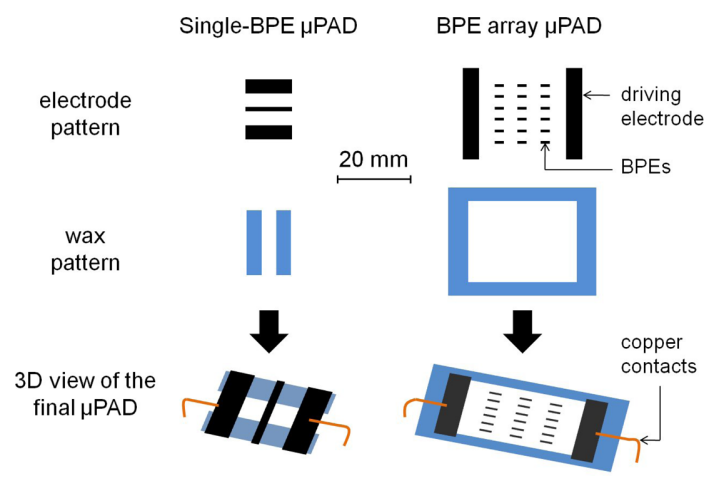

Fig. 1. Electrode and wax patterns used to fabricate a single-BPE $\mu \mathrm{PAD}$ and a BPE array $\mu \mathrm{PAD}$. The $3 \mathrm{D}$ views in the bottom row show the devices after screen printing the electrodes on the wax patterned paper. The black, blue, and white colors represent the carbon screen-printed electrodes, the wax, and the paper, respectively. The orange lines represent the copper tape used to connect the driving electrodes to a power supply.

nected to an external power supply using copper tape (Fig. 1). Note that the BPEs were always located between the two driving electrodes but were not connected to any external power source. During the ECL measurements, the $\mu$ PADs were sandwiched between two glass slides.

\subsection{Colorimetric measurements.}

The paper channel was soaked in a solution containing $10.0 \mathrm{mM}$ phosphate buffer $(\mathrm{pH} 7.6)$ and dried at $65^{\circ} \mathrm{C}$ for $1 \mathrm{~min}$. Then $8.0 \mu \mathrm{L}$ of a colorimetric $\mathrm{pH}$ indicator solution mixed with DI water $(30 \% \mathrm{v} / \mathrm{v})$ and $\mathrm{NaCl}(10.0 \mathrm{mM})$ was placed on the channel. The color of the channel instantly turned yellow-green indicating a pH of about 7-8. A DC power supply (TDKLAMBDA LLS9120, Tokyo, Japan) was used to power the device at $25.0 \mathrm{~V}$ for $90 \mathrm{~s}$.

\subsection{ECL measurements.}

The ECL intensity was recorded using a Nikon AZ100 microscope (0.5X Nikon AZ Plan Apo objective, no filter) equipped with a black and white Quantem 5125C camera. V++ Precision Digital Imaging software (Digital Optics, Auckland, New Zealand) was used to acquire the micrographs. An exposure time of $1 \mathrm{~s}$ was used for all micrographs. The light intensity emitted at the anodic poles of the BPEs was averaged over the surface of the BPE poles and background subtracted using Image J (NIH, Bethesda,

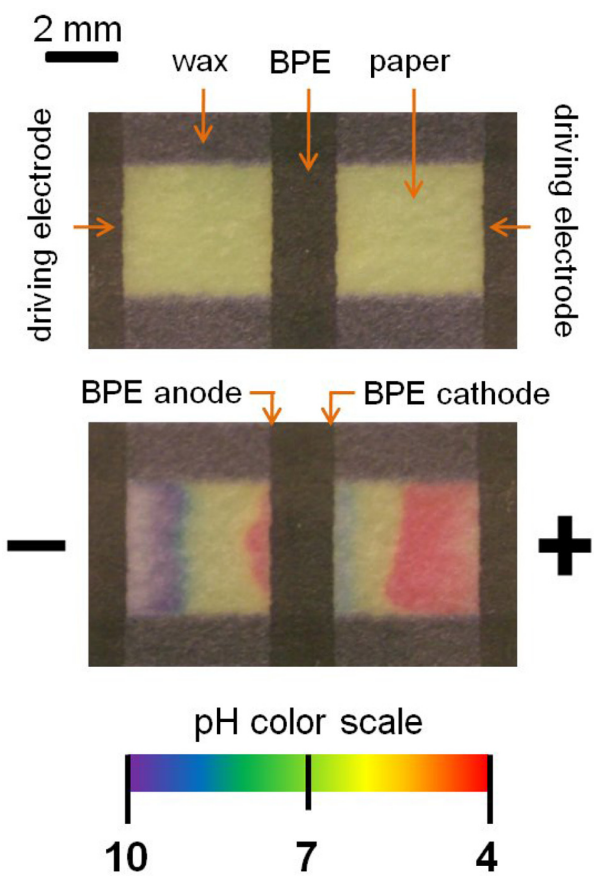

Fig. 2. Photographs of a single-BPE $\mu$ PAD. The top and bottom photographs were taken before and after application of $25.0 \mathrm{~V}$ for $90 \mathrm{~s}$, respectively. The channel was filled with a colorimetric $\mathrm{pH}$ indicator solution, which gives the yellowgreen coloration to the paper. The red and blue colors in the bottom photograph correspond to $\mathrm{pH}$ changes resulting from electrochemical reactions at the driving electrodes and the BPE.

MD). For the experiments shown in Fig. 5a, the time between measurements was $1 \mathrm{~min}$ to allow the rehomogenization of the ECL reagents in the diffusion layer. A DC power supply was used to power the paper device.

\section{Results and Discussion}

\subsection{Bipolar electrochemistry on a $\mu$ PAD}

The device shown in Fig. 2 was used for a proof-ofconcept experiment to demonstrate that bipolar electrochemistry can be carried out on a $\mu$ PAD using carbon SPEs. The figure shows two carbon driving electrodes screen-printed at the two opposing edges of a wax-patterned paper channel $(12.0 \mathrm{~mm}$ long by $4.5 \mathrm{~mm}$ wide). A $2.0 \mathrm{~mm}$ long carbon SPE is defined at the center of the paper channel and between the driving electrodes. To demonstrate that faradaic processes occur simultaneously at the poles of the BPE, an elegant colorimetric experiment, initially reported 
by Manz and co-workers, was carried out. ${ }^{18}$ The principles of this experiment are summarized in Scheme $1 \mathrm{~b}$. In the presence of a sufficiently large value of $E_{\text {tot }}$, water is oxidized and reduced at the anodic and cathodic poles of a BPE generating protons and hydroxide ions, respectively. A colorimetric $\mathrm{pH}$ indicator solution is used to show the resulting changes in the local $\mathrm{pH}$ of the electrolyte solution. The initial green coloration in the paper channel (Fig. 2, top) arises from the colorimetric $\mathrm{pH}$ indicator solution filling the channel. However, when a potential bias of $E_{\text {tot }}=25.0 \mathrm{~V}$ is applied between the two driving electrodes for $90 \mathrm{~s}$ (Fig. 2, bottom), the color of the dye changes near the edge of each electrode. At the positive driving electrode the color changes from green to red, indicating that the $\mathrm{pH}$ decreases (generation of $\mathrm{H}^{+}$), and near the negative driving electrode the color changes from green to purple, indicating an increase of the $\mathrm{pH}$ (generation of $\mathrm{OH}^{-}$). Most importantly, however, the solution-saturated paper near the cathodic and anodic poles of the central BPE turned from green to purple and green to red, corresponding to the evolution of $\mathrm{OH}^{-}$and $\mathrm{H}^{+}$, respectively, even in the absence of a direct electrical connection. This result correlates to the case depicted in Scheme $1 \mathrm{~b}$ and clearly demonstrates that bipolar electrochemistry can be performed on a $\mu$ PAD.

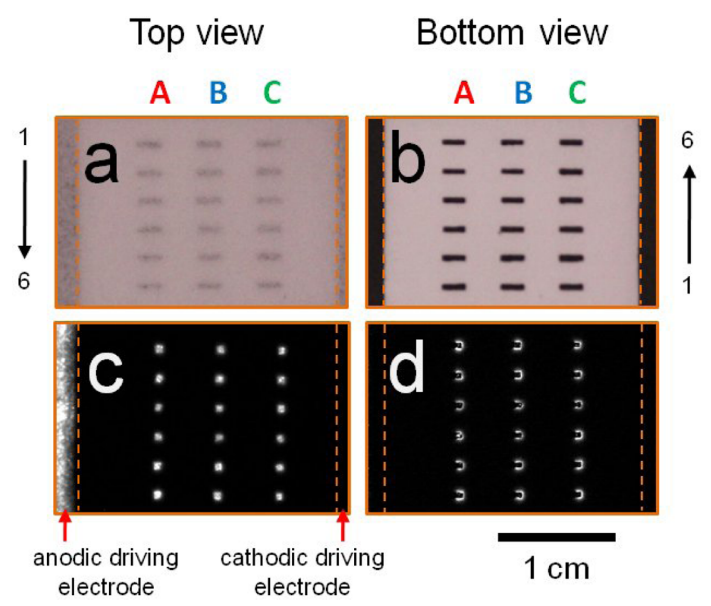

Fig. 3. (a, b) Optical and (c, d) luminescence micrographs of a $\mathrm{BPE}$ array $\mu \mathrm{PAD}$. The luminescence micrographs were obtained using $E_{\mathrm{tot}}=35.0 \mathrm{~V}$. The positions of the driving electrodes are indicated by the orange dashed line. $\left[\mathrm{Ru}(\mathrm{bpy})_{3}{ }^{2+}\right]=5.0 \mathrm{mM}$ and $[\mathrm{DBAE}]=25.0 \mathrm{mM}$ in $0.10 \mathrm{M}$ phosphate buffer (pH 6.9).

\subsection{ECL detection on a BPE array $\mu$ PAD}

As mentioned earlier, bipolar electrochemistry is well suited for multiplexed analyses, a functionality often desired in POC applications, and ECL provides a convenient and sensitive readout for large arrays of BPEs. ${ }^{19,31}$ To test the feasibility of using ECL-based readout for an array of paper-supported BPEs, a $\mu$ PAD composed of 18 individual BPEs was fabricated. The BPE array $\mu$ PAD was filled with $40.0 \mu \mathrm{L}$ of $0.10 \mathrm{M}$ phosphate buffer solution ( $\mathrm{pH}$ 6.9) containing $5.0 \mathrm{mM}$ $\mathrm{Ru}(\mathrm{bpy})_{3}{ }^{2+}$ and $25.0 \mathrm{mM}$ DBAE. ${ }^{32}$ Optical micrographs of the device are shown in Figs. $3 \mathrm{a}$ and $3 \mathrm{~b}$. Figs. $3 \mathrm{c}$ and $3 \mathrm{~d}$ show top and bottom luminescence micrographs, respectively, of the device after applying $E_{\text {tot }}=35.0 \mathrm{~V}$ to the driving electrodes. A strong ECL signal is apparent at the anodic pole of each of the $18 \mathrm{BPEs}$, as well as at the anodic driving electrode (only shown in Fig. 3c). Because wetted paper is semitransparent, and ECL is produced at the interface between the paper matrix and the electrodes, the strongest ECL signal is collected from the top of the device (that is, on the side of the paper opposite to the electrodes). However, lower intensity ECL can also be observed from the bottom of the $\mu$ PAD (Fig. 3d).

Luminescence micrographs of a BPE array $\mu$ PAD powered at different values of $E_{\text {tot }}$ are shown in Fig. 4a. The ECL intensity above the anodic driving electrode (left edge of the micrographs in Fig. 4a) does not vary significantly for values of $E_{\text {tot }}$ ranging from 20.0 to $35.0 \mathrm{~V}$. However, the ECL intensity observed at the BPEs increases dramatically as $E_{\text {tot }}$ increases. The average, normalized ECL intensity produced at the BPEs in the array, $I_{E C L}^{a v}$, is plotted in Fig. $4 \mathrm{~b}$ as a function of $E_{\text {tot }}$. These results show that a minimum $E_{\text {tot }}$ of $\sim 25.0 \mathrm{~V}$ is required to produce ECL. That is, at values of $E_{\text {tot }}>25.0 \mathrm{~V}, \Delta E_{\text {elec }}$, which is approximately linearly related to $E_{\text {tot }}$, ${ }^{21}$ is sufficient to simultaneously drive the oxidation of DBAE and Ru(bpy) ${ }_{3}{ }^{2+}$ (as well as the reduction of oxygen at the cathodic pole of the BPE) and hence ECL is observed. As shown in Fig. 4b, higher values of $\Delta E_{\text {elec }}$ lead to increased ECL intensity due to increased kinetics of the relevant faradaic reactions. $^{33}$

In our experiments, $E_{\mathrm{tot}}=35.0 \mathrm{~V}$ led to intense and reproducible ECL signals, and therefore this value was used for a quantitative study of ECL generated by the BPE array $\mu$ PADs. Figure 5a shows the ECL intensity, $I_{\mathrm{ECL}}$, obtained from 6 consecutive measurements of 6 of the 18 BPEs present on a single device. The coeffi- 
a

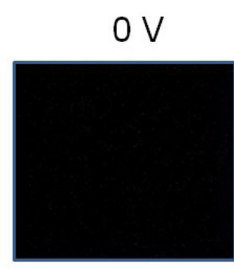

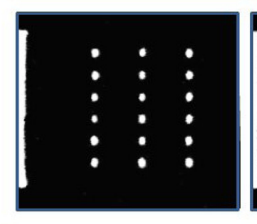

$25 \mathrm{~V}$

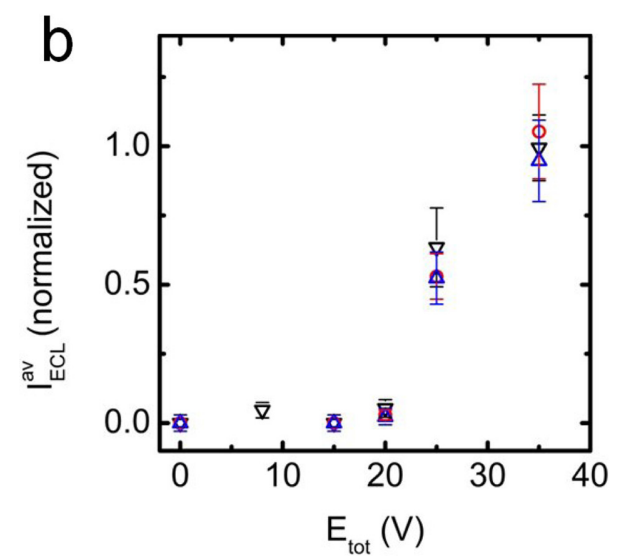

Fig. 4. (a) Luminescence micrographs of a BPE array $\mu \mathrm{PAD}$ operating at the indicated values of $E_{\mathrm{tot}}$. Note that a strong ECL signal is produced at the anodic driving electrode (on the left side of 3 of the micrographs), indicating that the device is operating correctly. The brightness and contrast of the micrographs have been adjusted to distinguish the ECL signal from the background. (b) Normalized ECL intensity averaged over the 18 BPEs present in a single device as a function of $E_{\mathrm{tot}}$. The different colors correspond to measurements for three independently prepared devices. The error bars correspond to the SD of the ECL intensity between BPEs on the same array.

cient of variation (CV, defined as the ratio of the standard deviation, SD, divided by the average intensity) of $I_{\mathrm{ECL}}$ for the six consecutive measurements is $4 \%$. This small variation in $I_{\mathrm{ECL}}$ means that a single device can be read out several times without loss of accuracy or reproducibility.

The variation of $I_{\mathrm{ECL}}$ for BPEs on the same array (intra-array variability) can be estimated from Fig. $5 \mathrm{~b}$. Here, the value of $I_{\mathrm{ECL}}$ is plotted as a function of the position of the BPE in the array. The numbering of the
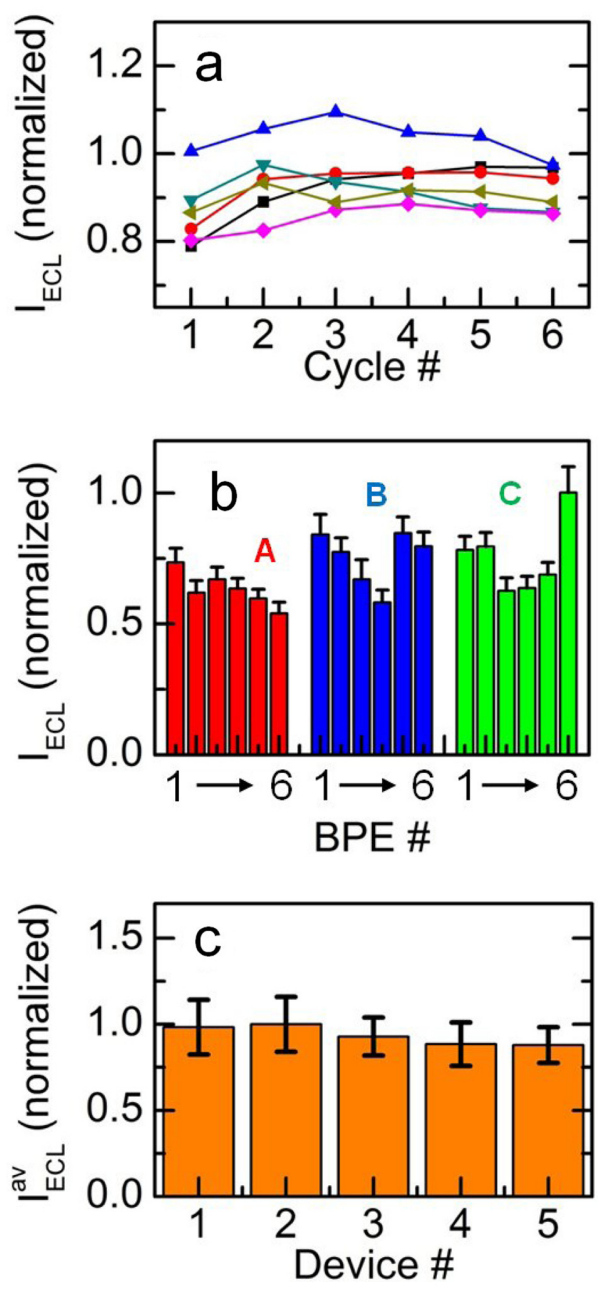

Fig. 5. (a) Six successive measurements of the ECL intensity (normalized) of 6 of the 18 BPEs present on a single BPE array $\mu$ PAD. An equilibration time of $1 \mathrm{~min}$ was allowed between each measurement. (b) Normalized ECL intensity at each BPE of a single BPE array $\mu$ PAD as a function of the position of the BPE in the array. The number and color coding of the BPEs is the same as in Fig. 3. The error bars represent the SD for three successive measurements. (c) Average ECL intensities obtained using five independently prepared BPE array $\mu$ PADs. The error bars represent the SD of $I_{E C L}^{a v}$.

BPEs follows the color and letter code shown in Fig. 3. The CV (defined in this case as the ratio of the SD of three successive measurements divided by $I_{E C L}^{a v}$ ) of the intra-array ECL intensity is $16 \%$. This value is adequate for manually-produced SPEs but would likely be improved by more reproducible fabrication processes. 
To compare reproducibility between devices (interarray variability), the average intensity per array was measured using 5 different BPE array $\mu$ PADs (Fig. 5c). The $\mathrm{CV}$ was calculated to be $6 \%$. This low $\mathrm{CV}$ value indicates good reproducibility between $\mu$ PADs, a crucial point for sensing applications. Note that although the inter-array CV is lower than the intraarray $\mathrm{CV}$, this is likely a mathematical artifact caused by averaging over a larger number of samples (18 $\mathrm{BPEs} /$ device) for the inter-array $\mathrm{CV}$.

\section{Conclusion}

To summarize, we have shown that carbon electrodes screen-printed directly onto cellulose paper can be used as BPEs. By using an array consisting of 18 individual BPEs, we also showed that ECL can be generated simultaneously at each BPE. Importantly, an $E_{\text {tot }}$ of $35.0 \mathrm{~V}$ was sufficient to reproducibly drive the ECL reaction. The reproducibility between individual BPEs and between devices is relatively good and demonstrates that ECL can be used as an optical reporting method on paper substrates. More importantly, because each BPE in the array does not require a direct electrical connection, a single power supply connected to a single pair of driving electrodes is sufficient to power a BPE array of arbitrary size. Accordingly, arrays such as these, fabricated on paper, could be used to simultaneously detect a large number of analytes, thereby opening the way to densely multiplexed $\mu$ PADs.

\section{Acknowledgments}

This project is sponsored by the Department of the Defense, Defense Threat Reduction Agency (contract number HDTRA-1-13-1-0031). RMC thanks the Robert A. Welch Foundation (Grant F-0032) for sustained research support.

\section{References}

1. Martinez, A. W.; Phillips, S. T.; Butte, M. J.; Whitesides, G. M. Patterned Paper as a Platform for Inexpensive, LowVolume, Portable Bioassays. Angew. Chem., Int. Ed., 46, 1318 (2007).

2. Martinez, A. W.; Phillips, S. T.; Whitesides, G. M.; Carrilho, E. Diagnostics for the Developing World: Microfluidic Paper-Based Analytical Devices. Anal. Chem., 82, 3 (2010)
3. Parolo, C.; Merkoci, A. Paper-based nanobiosensors for diagnostics. Chem. Soc. Rev., 42, 450 (2013).

4. Yetisen, A. K.; Akram, M. S.; Lowe, C. R. Paper-based microfluidic point-of-care diagnostic devices. Lab Chip, 13, 2210 (2013).

5. Li, X.; Ballerini, D. R.; Shen, W. A perspective on paperbased microfluidics: Current status and future trends. Biomicrofluidics, 6, 11301 (2012).

6. Lewis, G. G.; DiTucci, M. J.; Phillips, S. T. Quantifying Analytes in Paper-Based Microfluidic Devices Without Using External Electronic Readers. Angew. Chem., Int. Ed., 51, 12707 (2012).

7. Scida, K.; Li, B.; Ellington, A. D.; Crooks, R. M. DNA Detection Using Origami Paper Analytical Devices. Anal. Chem., 85, 9713 (2013).

8. Dungchai, W.; Chailapakul, O.; Henry, C. S. Electrochemical Detection for Paper-Based Microfluidics. Anal. Chem., 81, 5821 (2009).

9. Liu, H.; Xiang, Y.; Lu, Y.; Crooks, R. M. Aptamer-Based Origami Paper Analytical Device for Electrochemical Detection of Adenosine. Angew. Chem., Int. Ed., 51, 6925 (2012).

10. Santhiago, M.; Wydallis, J. B.; Kubota, L. T.; Henry, C. S. Construction and Electrochemical Characterization of Microelectrodes for Improved Sensitivity in Paper-Based Analytical Devices. Anal. Chem., 85, 5233 (2013).

11. Glatzel, S.; Schnepp, Z.; Giordano, C. From Paper to Structured Carbon Electrodes by Inkjet Printing. Angew. Chem., Int. Ed., 52, 2355 (2013).

12. Tobjörk, D.; Österbacka, R. Paper electronics. Adv Mater, 23, 1935 (2011).

13. Maxwell, J. E.; Mazzeo, A. D.; Whitesides, G. M. Paperbased electroanalytical devices for accessible diagnostic testing. MRS Bull., 38, 309 (2013).

14. Nie, Z.; Deiss, F.; Liu, X.; Akbulut, O.; Whitesides, G. M. Integration of paper-based microfluidic devices with commercial electrochemical readers. Lab Chip, 10, 3163 (2010).

15. Wang, P.; Ge, L.; Yan, M.; Song, X.; Ge, S.; Yu, J. Paperbased three-dimensional electrochemical immunodevice based on multi-walled carbon nanotubes functionalized paper for sensitive point-of-care testing. Biosens. Bioelectron., 32, 238 (2012).

16. Wu, Y.; Xue, P.; Kang, Y.; Hui, K. M. Paper-Based Microfluidic Electrochemical Immunodevice Integrated with Nanobioprobes onto Graphene Film for Ultrasensitive Multiplexed Detection of Cancer Biomarkers. Anal. Chem., 85, 8661 (2013).

17. Delaney, J. L.; Hogan, C. F.; Tian, J.; Shen, W. Electrogenerated Chemiluminescence Detection in PaperBased Microfluidic Sensors. Anal. Chem., 83, 1300 (2011).

18. Fosdick, S. E.; Knust, K. N.; Scida, K.; Crooks, R. M. Bipolar Electrochemistry. Angew. Chem., Int. Ed., 52, 10438 (2013).

19. Chow, K.-F.; Mavré, F.; Crooks, J. A.; Chang, B.-Y.; 
Crooks, R. M. A Large-Scale, Wireless Electrochemical Bipolar Electrode Microarray. J. Am. Chem. Soc., 131, 8364 (2009).

20. Fosdick, S. E.; Berglund, S. P.; Mullins, C. B.; Crooks, R. M. Parallel Screening of Electrocatalyst Candidates Using Bipolar Electrochemistry. Anal. Chem., 85, 2493 (2013).

21. Mavré, F.; Anand, R. K.; Laws, D. R.; Chow, K.-F.; Chang, B.-Y.; Crooks, J. A.; Crooks, R. M. Bipolar Electrodes: A Useful Tool for Concentration, Separation, and Detection of Analytes in Microelectrochemical Systems. Anal. Chem., 82, 8766 (2010).

22. Bradley, J.-C.; Ma, Z. Contactless Electrodeposition of Palladium Catalysts. Angew. Chem., Int. Ed., 38, 1663 (1999).

23. Klett, O.; Nyholm, L. Separation High Voltage Field Driven On-Chip Amperometric Detection in Capillary Electrophoresis. Anal. Chem., 75, 1245 (2003).

24. Ordeig, O.; Godino, N.; del Campo, J.; Muñoz, F. X.; Nikolajeff, F.; Nyholm, L. On-Chip Electric Field Driven Electrochemical Detection Using a Poly(dimethylsiloxane) Microchannel with Gold Microband Electrodes. Anal. Chem., 80, 3622 (2008).

25. Chow, K.-F.; Chang, B.-Y.; Zaccheo, B. A.; Mavré, F.; Crooks, R. M. A Sensing Platform Based on Electrodissolution of a $\mathrm{Ag}$ Bipolar Electrode. J. Am. Chem. Soc., 132, 9228 (2010).

26. Fosdick, S. E.; Crooks, R. M. Bipolar Electrodes for Rapid Screening of Electrocatalysts. J. Am. Chem. Soc., 134, 863
(2011).

27. Zhan, W.; Crooks, R. M. Microelectrochemical Logic Circuits. J. Am. Chem. Soc., 125, 9934 (2003).

28. Chow, K.-F.; Mavré, F.; Crooks, R. M. Wireless Electrochemical DNA Microarray Sensor J. Am. Chem. Soc., 130, 7544 (2008).

29. Lu, Y.; Shi, W.; Jiang, L.; Qin, J.; Lin, B. Rapid prototyping of paper-based microfluidics with wax for low-cost, portable bioassay. Electrophoresis, 30, 1497 (2009).

30. Carrilho, E.; Martinez, A. W.; Whitesides, G. M. Understanding Wax Printing: A Simple Micropatterning Process for Paper-Based Microfluidics. Anal. Chem., 81, 7091 (2009).

31. Deiss, F. d. r.; LaFratta, C. N.; Symer, M.; Blicharz, T. M.; Sojic, N.; Walt, D. R. Multiplexed Sandwich Immunoassays Using Electrochemiluminescence Imaging Resolved at the Single Bead Level. J. Am. Chem. Soc., 131, 6088 (2009).

32. Sun, S.; Yang, Y.; Liu, F.; Pang, Y.; Fan, J.; Sun, L.; Peng, X. Study of Highly Efficient Bimetallic Ruthenium Trisbipyridyl ECL Labels for Coreactant System. Anal. Chem., 81, 10227 (2009).

33. Mavré, F.; Chow, K.-F.; Sheridan, E.; Chang, B.-Y.; Crooks, J. A.; Crooks, R. M. A Theoretical and Experimental Framework for Understanding Electrogenerated Chemiluminescence (ECL) Emission at Bipolar Electrodes. Anal. Chem., 81, 6218 (2009). 